International Journal of Instrumentation and Control Systems (IJICS) Vol.8, No.1, January 2018

\title{
FURTHER RESEARCH ON THE QUADRANGULAR PRISM OF SELF-INVISIBILITY (II)
}

\author{
Yizong $\mathrm{He}^{*}$
}

\author{
Department of Electrical and Computer Engineering, University of Minnesota, \\ Minneapolis, MN 55455, USA \\ yizonghe@126. com
}

\begin{abstract}
A kind of novel pillar (used to support a windshield) has been designed to replace the conventional ones used in all sorts of vehicles. The prominent advantage of such a pillar is that it appears invisible so that the blind spots caused by $A, B, C$ and $D$ pillars will be diminished to a large extent or even eliminated completely. Therefore, the driver's view is enlarged considerably. An intrinsic disadvantage of the new pillar, directionality and the invisibility rate with respect to the deviation from most-clear position, were investigated quantitatively and discussed.
\end{abstract}

\section{KEYWORDS}

stealth technology, camouflage technology, vehicle, blind spot, A pillar automobile industry

\section{INTRODUCTION}

It is well known that camouflage technology has been researched and widely used in military industry, especially in aircrafts [1-3]. Instead of reflecting or absorbing electromagnetic waves radiated from radar, the second category of stealth research is concerned with how to bend the visible light bouncing from the objects camouflaged [4-6]. However, because of security and confidentiality in stealth technology, until now there is rarely any application of stealth technology in daily life.

As part of the basic and essential structure of a car, A-pillars frame the windshield and absorb the considerable energy of a crash caused by a traffic accident. On the other hand, A pillars inevitably produce blind spots on each side of a car, with other blind spots near the side mirrors. If the pillars inside any vehicle can be camouflaged, the driver can obtain a wider view. There is no doubt camouflage technology for car pillars is a significant research area as it is in military industry.

So much effort has been made to reduce down the interference caused by the A, B, C and D pillars inside a vehicle. Several approaches are listed as follows:

First, in the Audi car, two A-pillars have been removed directly [7]. It did widen the driver's sightlines perfectly, but one surely suspects that such an improvement gives rise to the problem of

DOI : 10.5121/ijics.2018.8101 
International Journal of Instrumentation and Control Systems (IJICS) Vol.8, No.1, January 2018

security because the two A-pillars actually play an essential role in resisting collision energy and rollover, in addition to framing the windshield and supporting the roof.

Second, installing monitors to supervise the area blocked by two A pillars and forming an image on two small monitors embedded inside two A pillars[8]. Evidently, a proportion of A pillars is still covered by the screen, so visibility is still impaired.

Third, the Volvo Safety car has changed conventional A pillars into ones with triangular windows, so that the two A-pillars become partially transparent [9]. However, the skeleton of the pillars still hamper some of the driver's sightlines.

Fourth, on one hand, research on new A-pillars focused on enhancing safety has been reported [10]. In order to meet the new roof-crush standards, ultra-high-strength steel should be used for A-pillars since a vehicle must withstand a force equal to four times its weight before the roof crushes $(12.7 \mathrm{~cm})$. However, on the other hand, A-pillars must be soft enough to meet federal head-impact standards. Consequently, the size of A-pillars will inevitably grow significantly larger. At the same time A-pillar blind spots will obscure more sightlines of the driver. How can designers enhance safety without impeding visibility?

In this paper, a novel device, a quadrangular prism of self-invisibility, was tried to replace the conventional A, B, C and D pillars, and pertinent invisibility rate with respect to the deviation of observer point from the most-clear position was investigated quantitatively $[11,12]$.

\section{RePlacing CONVENTIONAL A, B, C AND D Pillars With INVISIBLE QUADRANGULAR PRISMS}

Several pillars, A, B, C and D pillars, serve to frame the windshield, side windows and top roof of a vehicle. Thus the issue is how to camouflage all the pillars shown in Fig.1, 2, 3 and 4. The two main pillars influencing the driver's sightlines are the A-pillars. Therefore, the first attempt is to replace A-pillars with the new invisible device (see Fig. 5). In order to get the best invisible effect, except for the quadrangular prism, all the other components shown in Fig. 5 should be transparent substances serving to buffer the possible collision and inevitable vibration of the windshield and all the side glass windows. Since the incident light should be reflected by lateral surface mirrors of triangular prisms, the outside surface of new pillars should be kept quite clean, so that the reflection coefficient can be close to 1 . So the transparent covers (5) in Fig. 5 are necessary.

As mentioned before [12], an intrinsic disadvantage of this invisible quadrangular prism is the existence of directionality. In light of different statures of drivers, the deviation and the separation between the observer's eyes and the quadrangular prisms will inevitably vary to some extent. Consequently, investigating the invisibility rate as the function of separation and deviation is essential for the design of an invisible quadrangular prism. The invisibility rate with respect to deviation along y axis has been calculated quantitatively [12]. Similarly, in Fig. 7, which is an abstract model from Fig. 6, suppose $\mathrm{OF}=1$ meter, $\mathrm{EF}=0.2$ meter, $\mathrm{AB}=0.48$ meter, $\mathrm{CD}=0.40$ meter, accordingly, the coordinates of point $\mathrm{A}, \mathrm{B}, \mathrm{C}, \mathrm{D}$ are as following: $\mathrm{A}(0.24,1.2), \mathrm{B}(-0.24$, 1.2), $\mathrm{C}(0.20,1), \mathrm{D}(-0.20,1), \mathrm{Q}(\mathrm{x}, 0), \mathrm{P}(\mathrm{xo}, 0)$, respectively. Thus the following equations can be gotten. 
International Journal of Instrumentation and Control Systems (IJICS) Vol.8, No.1, January 2018

$$
\begin{aligned}
& \alpha=\mathrm{AQD}=\angle \mathrm{AQO}-\angle D Q O \\
& \beta=\angle \mathrm{CQB}=\angle \mathrm{CQO}-\angle B Q O \\
& 0<\mathrm{x}<0.20 \\
& \alpha=\pi-\arctan \frac{1.2}{0.24-x}-\arctan \frac{1}{x+0.20} \\
& \beta=\pi-\arctan \frac{1}{0.20-x}-\arctan \frac{1.2}{x+0.24} \\
& 0.20<\mathrm{x}<0.24 \\
& \alpha=\pi-\arctan \frac{1.2}{0.24-x}-\arctan \frac{1}{x+0.20} \\
& \beta=\pi-\arctan \frac{1}{x-0.20}-\arctan \frac{1.2}{x+0.24} \\
& \mathrm{x}>0.24 \\
& \alpha=\arctan \frac{1.2}{x-0.24}-\arctan \frac{1}{x+0.20}-\cdots \\
& \beta=\arctan \frac{1}{x-0.20}-\arctan \frac{1.2}{x+0.24}
\end{aligned}
$$

Table 1 shows the outcome of the invisibility rate with respect to the deviation along $\mathrm{x}$ axis. This graph is shown in Fig. 8. Obviously, the invisibility rate goes down in a nearly linear way as the deviation increases.

\section{DISCUSSION AND CONCLUSION}

So far, this brief discussion focused only on A-pillars. In fact, all other pillars should be camouflaged in a similar way. B and C-pillars, directly cause part of rear mirror blind spots. It seems D-pillars do not influence driver's sightlines, because the driver rarely turns to look back behind the car. However, D-pillars do impede the driver's view because they form their images visible in the inside rearview mirror.

The directionality problem seems essential for such an invisible system. Fortunately the invisibility rate still reaches to $60.06 \%$ even when the deviation equals to $60 \%$ of OF along $\mathrm{x}$ axis. Therefore, such a quadrangular prism of self-invisibility meet the practical situation varying according to driver's stature which causes the deviation away from most-clear position. Based on the above investigation, a definite conclusion can be obtained that quadrangular prisms are promising to serve as invisible pillars to replace conventional ones that frame the windshield, side windows, and support the roof of vehicles. More research concerning the mechanical strength of the new pillars to absorb the energy of collision is underway. 
International Journal of Instrumentation and Control Systems (IJICS) Vol.8, No.1, January 2018
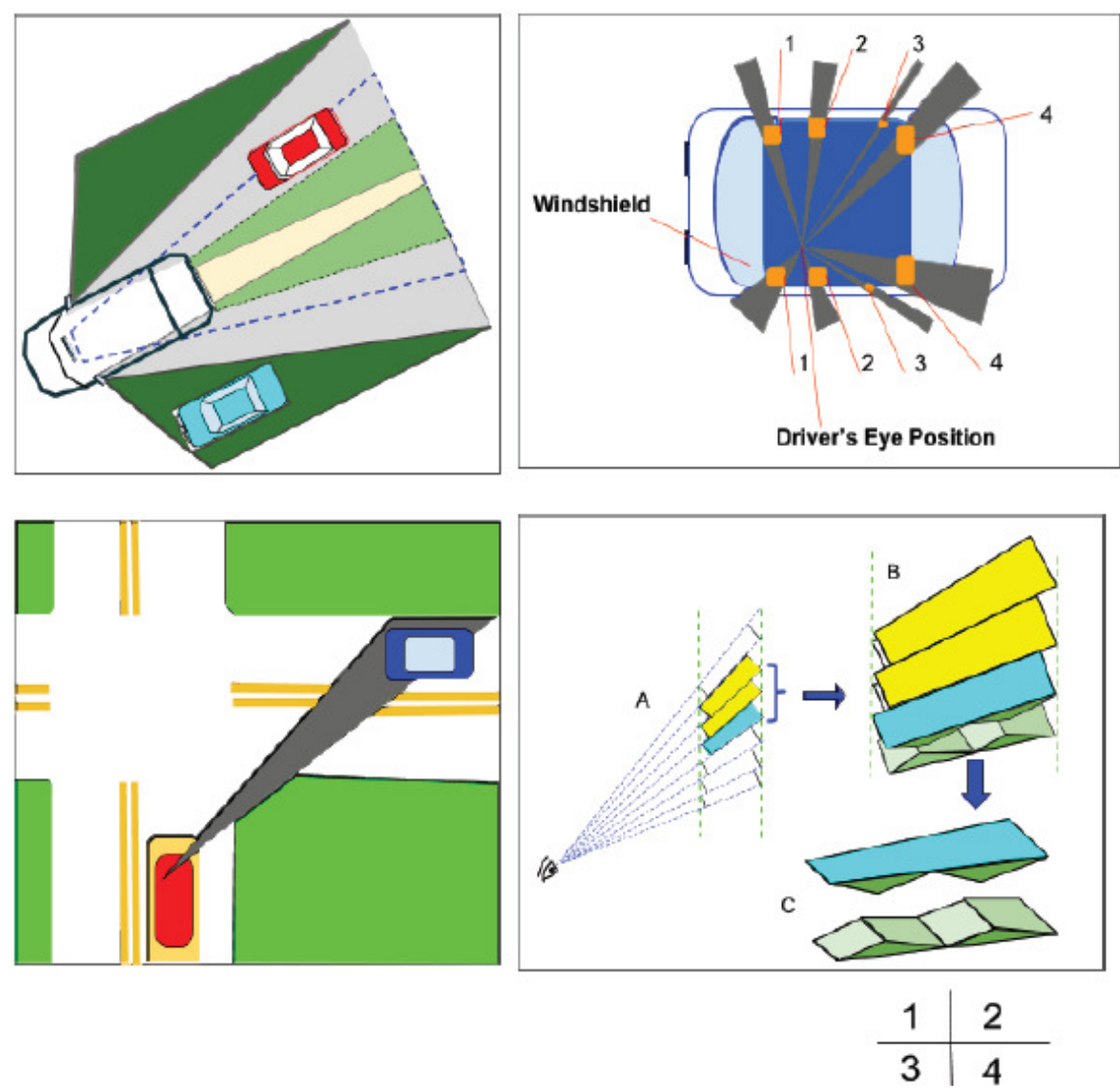

Fig. 1 Blind spots exist in a wide range of vehicles. The driver of the white car sees the red car through the mirrors, but cannot see the blue car without turning to check his blind spot.

Fig. 2 Illustration of the pillars supporting the windshield which causes blind spots. 1, 2, 3 and 4 represent, A, B, C and D-pillars, respectively

Fig. 3 Illustration of blind spots caused by A pillars.

Fig. 4 Illustration of a quadrangular prism of self-invisibility used to replace pillars.

Fig. 5 Illustration of a new invisible, quadrangular prism to replace conventional right A-pillar of vehicle. (1) windshield; (2) side window; (3) transparent cushion; (4) a quadrangular prism of selfinvisibility; (5) transparent cover; (6) transparent soft stuffed material.

Fig. 6 Sectional graph of the quadrangular prism of self-invisibility

Fig. 7 Deviation away from most-clear position of the abstract model of a quadrangular prism of selfinvisibility shown in Fig. 6

Fig. 8 The invisibility rate goes down when the observation point deviates away from most-clear position along $\mathrm{x}$ axis 
International Journal of Instrumentation and Control Systems (IJICS) Vol.8, No.1, January 2018
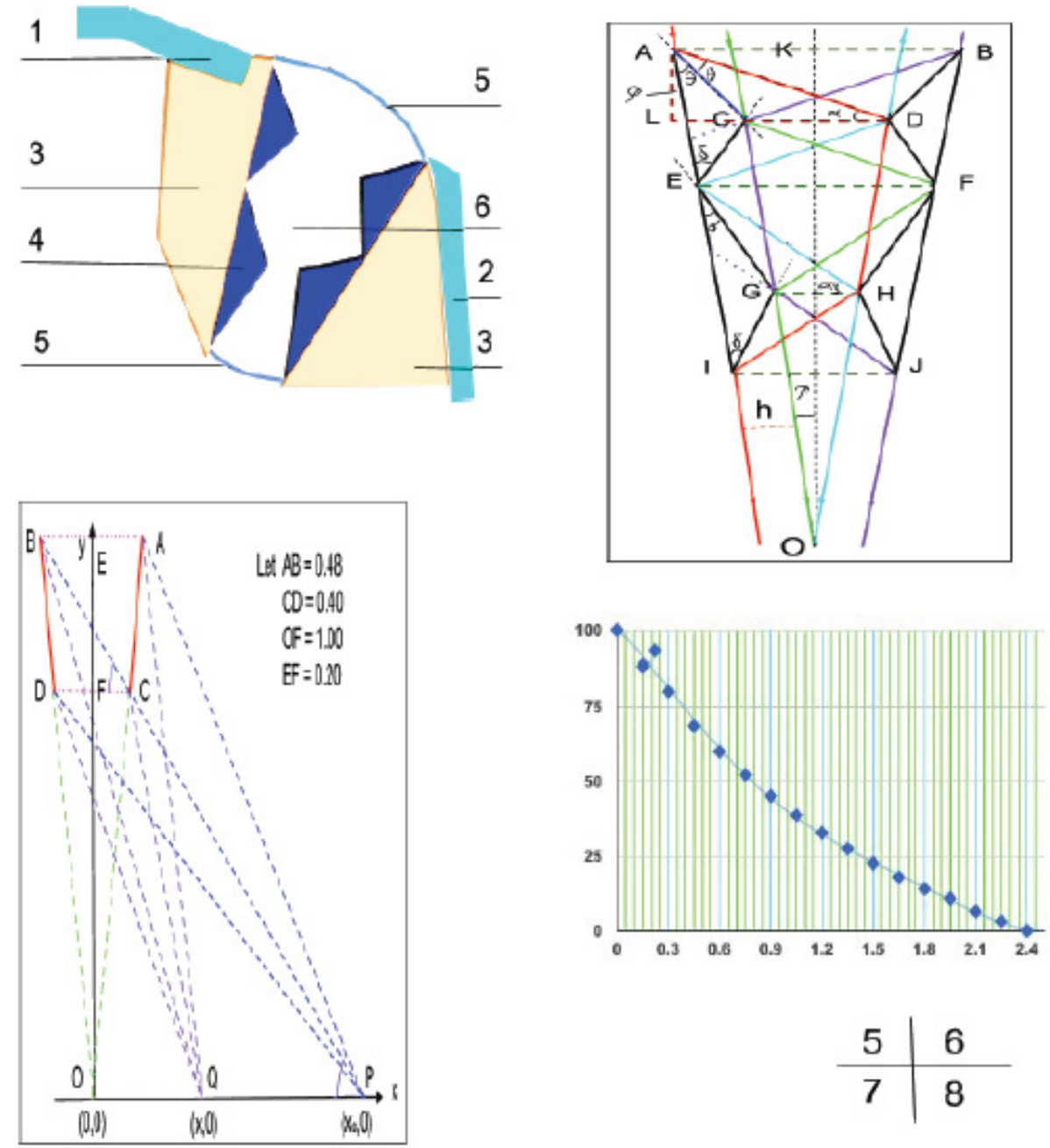

Table 1 The invisibility rate vs. deviation away from most-clear position along $\mathrm{x}$ axis of the quadrangular prism of self-invisibility

\begin{tabular}{|l|l|l|l|l|l|l|l|l|l|}
\hline$x$ & 0.15 & 0.22 & 0.30 & 0.45 & 0.60 & 0.75 & 0.90 & 1.05 & 1.20 \\
\hline$\alpha$ & 23.58 & 23.74 & 23.71 & 23.10 & 21.96 & 20.50 & 18.92 & 17.32 & 15.80 \\
\hline$\beta$ & 20.86 & 22.12 & 18.52 & 15.86 & 13.19 & 10.71 & 8.54 & 6.71 & 5.19 \\
\hline$\eta=\frac{\beta}{a}$ & 88.46 & 93.22 & 80.17 & 68.66 & 60.06 & 52.24 & 45.14 & 38.74 & 32.85 \\
\hline$x$ & 1.35 & 1.50 & 1.65 & 1.80 & 1.95 & 2.10 & 2.25 & 2.40 & \\
\hline$\alpha$ & 14.4 & 13.13 & 12.14 & 11.00 & 10.12 & 9.33 & 8.54 & 8.01 & \\
\hline$\beta$ & 3.97 & 2.98 & 2.18 & 1.55 & 1.02 & 0.61 & 0.27 & 0 & \\
\hline$\eta=\frac{\beta}{a}$ & 27.57 & 22.67 & 17.96 & 14.09 & 10.8 & 6.54 & 3.16 & 0 & \\
\hline
\end{tabular}


International Journal of Instrumentation and Control Systems (IJICS) Vol.8, No.1, January 2018

\section{REFERENCES AND LINKS}

[1] http://www.hyperstealth.com/Quantum-Stealth/

[2] Rao, G.A., \& Mahulikar, S.P.: (2002) "Integrated review of stealth technology and its role in airpower", Aeronautical Journal, v. 106(1066): 629-641.

[3] Wenshan Cai, Uday K, Chenttiar, etc, Optical cloaking with metamaterisal, nature photonics, vol.1, April 2007

[4] Christof Koch, Rendering the visible in visible, Scientific American mind, oct/nov. 2008

[5] Hongsheng Chen, etc, Ray-optics cloaking devices for large objects in incoherent natural light, NATURE COMMUNICATIONS | 4:2652

[6] Vivek Angoth, Amarjot Singh, M. Sai Shanka, A Novel Refractive Technique for Achieving Macroscopic Invisibility of Visual Light, Physics and Materials Chemistry, Vol. 1, No. 1, 5-8, 2013

[7] http://auto.ifeng.com/buycar/leadbuy/20090408/23402.shtml

[8] http://fixyourcar.net/pillar-blind-spots/

[9] http://www.team-bhp.com/forum/indian-car-scene/91225-blind-spots-new-cars-roadView-driveability.html

[10] http://wardsauto.com/news-analysis/new-pillars-enhance-safety-impede-visibility

[11] Yizong He, A quadrangular prism of self-invisibility, International Journal of Instrumentation and Control Systems (IJICS) Vol.7, No.3, July 2017

[12] Yizong He, Further research on the quadrangular prism of self invisibility (I), International Journal of Instrumentation and Control Systems, Vol.7, No.4, October 2017. 